\title{
Experimental Verification of The Spot of Near-field Fresnel Zone Plate With Sweeping Frequency

\author{
Jian Zhao ${ }^{1, ~ a}$, Honghao Wei ${ }^{2, b}$
}

${ }^{1}$ School of Electronic and Information Engineering, Beihang University, Beijing, China

${ }^{2}$ School of Electronic and Information Engineering, Beihang University, Beijing, China

azhaojian6833@foxmail.com, bwhhbuaa@foxmail.com

Keywords: Fresnel zone plate, near-field, spot, sweeping frequency, experimental verification.

Abstract. The spot of near-field Fresnel zone plate (NFFZP) is very important in electromagnetic field. In this paper, an NFFZP which has been designed to focus a spherical wave at a spot is measured, and the formula for the steered near-field spot of the NFFZP versus frequency is presented and proved. Furthermore, the $\mathrm{W}_{3 \mathrm{~dB}}$ and sidelobe level (SLL) of the spot are characterized. All the results are verified by full-wave simulations and experiments in Ku-band.

\section{Introduction}

Focused spots are of great importance to microwave wireless power transmission, remote sensing and medical imaging [2, 3, 4]. The Fresnel zone plate (FZP) is an iportant spot generator because it has the advantage of being lighter and more easily designed and manufactured compared to the array antennas and reflectors. Moreover, FZP lenses are thinner than traditional lens antennas especially when a large antenna aperture is needed. In this paper, we study the properties of focal spot and present experimental verification of the formulas for the steered near-field spot of the NFFZP versus frequency.

\section{Concept and formulations}

For completeness, we offer a brief conceptual overview of the formulas for the steered near-field spot of the NFFZP versus frequency, which has already been thoroughly described in [1]. The feed is located at $\left(0,0, F_{1}\right)$, and the focal spot is at $\left(0,0, F_{2}\right)$. The normalized phase of the rays can be written as follows:

$\phi(r)=\frac{2 \pi f}{c}\left(\sqrt{r^{2}+F_{1}^{2}}+\sqrt{r^{2}+F_{2}^{2}}-F_{1}-F_{2}\right)$

where $f$ is the frequency, $c$ is the speed of light in free space, and $r(x, y, 0)$ describes the position of the secondary wavesource in the NFFZP.

Under the paraxial condition, (1) can be rewritten approximately as

$\phi(r) \cong \frac{2 \pi r^{2}}{c} \frac{f}{2}\left(\frac{1}{F_{1}}+\frac{1}{F_{2}}\right)-\frac{\pi}{c} \frac{f}{4}\left(\frac{r^{4}}{F_{1}^{3}}+\frac{r^{4}}{F_{2}^{3}}\right)+\mathrm{L}$

where the higher-order items are neglected, and the Fresnel approximation can be used [5]. For a manufactured FZP and the determined zones, while neglecting the higher-order items, the constrained relationships are formulated approximately as follows:

$\frac{1}{F_{1}}+\frac{1}{F_{2}} \propto \frac{1}{f}$

For the same configuration above the NFFZP and the fixed feeding position with a sweeping frequency $f^{\prime}$ according to (3), the new focused spots are steered at

$F_{2}^{\prime}=\frac{f^{\prime}}{f} \frac{F_{1} F_{2}}{F_{1}+\left(1-\frac{f^{\prime}}{f}\right) F_{2}}$ 
From the nonlinear (4), we can observe that the spot is displaced in a forward direction from the original focus when the frequency is higher than the centre frequency. The formula 4 is the formula for the steered near-field spot of the NFFZP versus frequency.

\section{Experimental Results}

Experimental testing platform is showed in figure 1. The port 1 of an Agilent E8357A Performance Network Analyzer (PNA) drove an Ku-band feed horn, transmitting a spherical wave with the E-plane aligned in the y-direction; this wave was collimated by a NFFZP len to form a spot which is detected by a probe connecting to port 2 of the PNA. The probe is fixed on the scanning frame and is moved by controling the scanning frame.

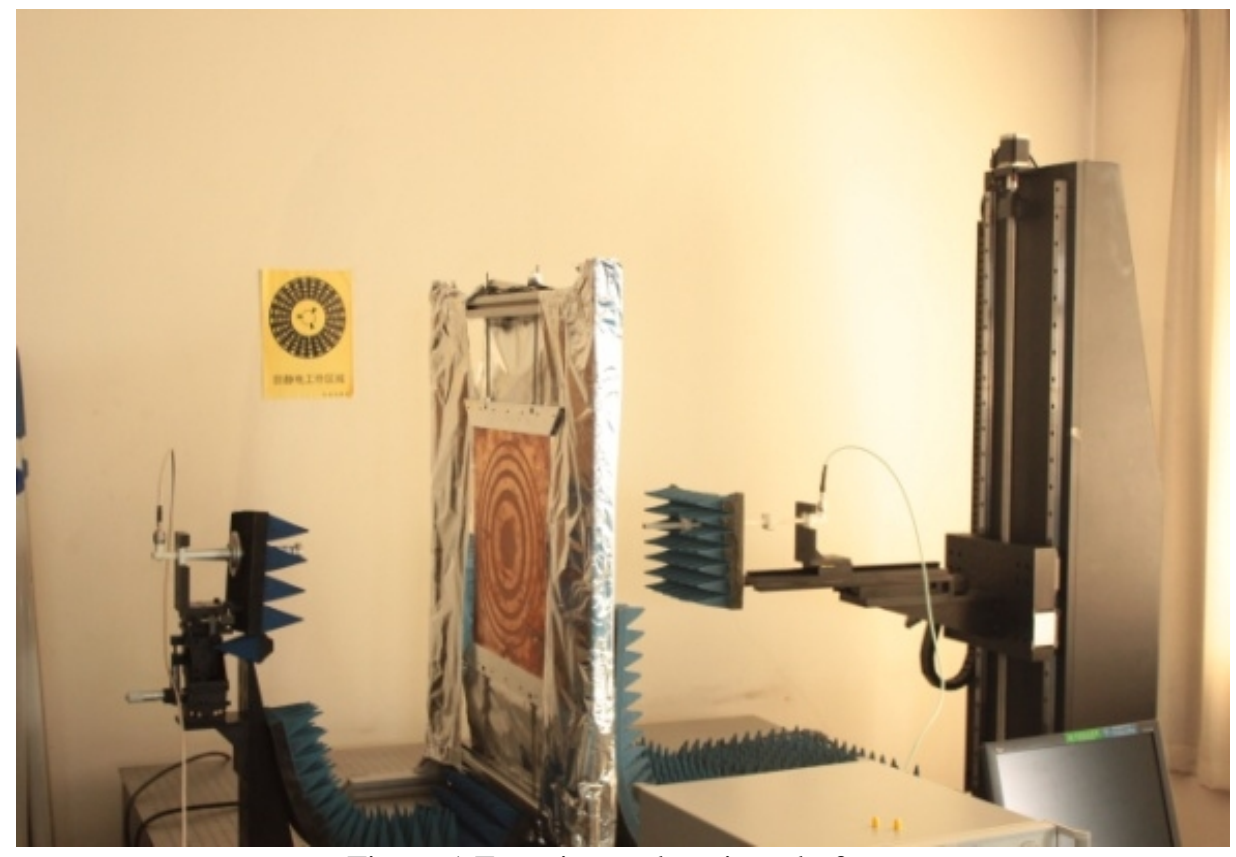

Figure 1 Experimental testing platform

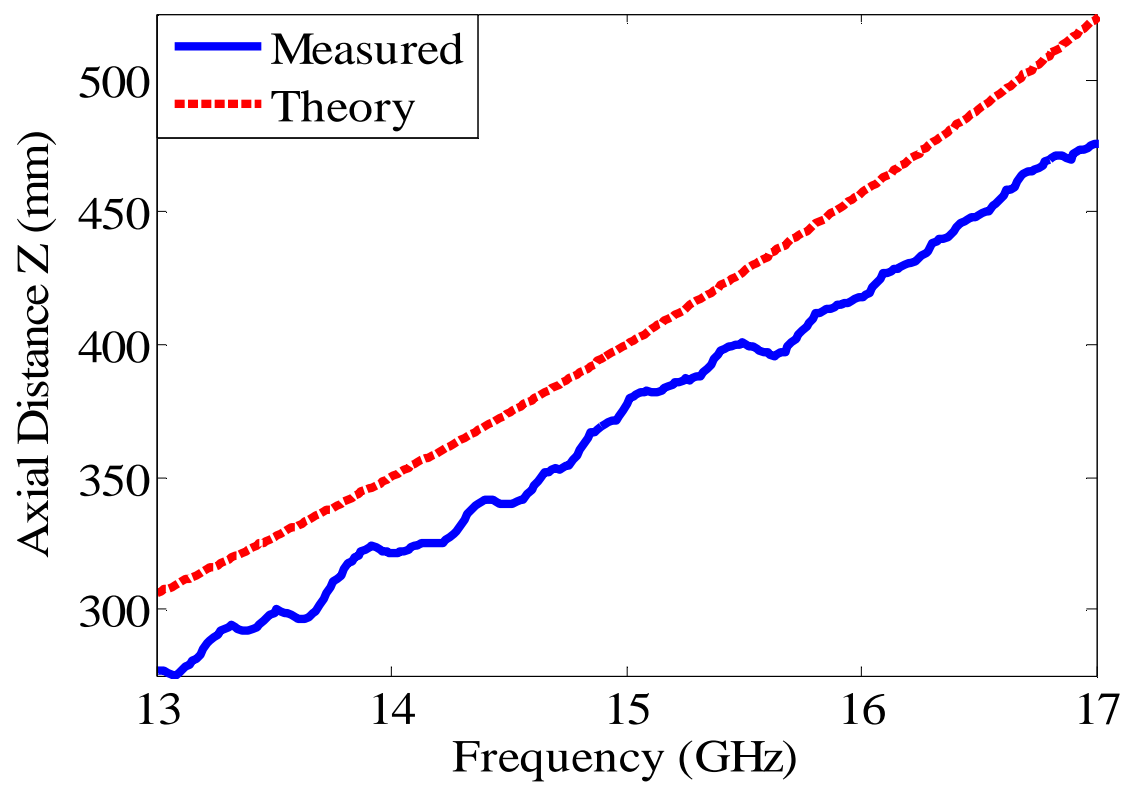

Figure 2 the curve of the steered near-field spot versus frequency

Figure 2 shows the formula for the steered near-field spot of the NFFZP versus frequency.The red dash-dotted line is getted by theoretical derivation. The blue line is getted by experiment. We can see that the theory derivation of the steered near-field spot of the NFFZP versus frequency is consistent with 
the experiment. Therefore,the formula 4 correctly reflects the movement curve of the focal spot with frequency.
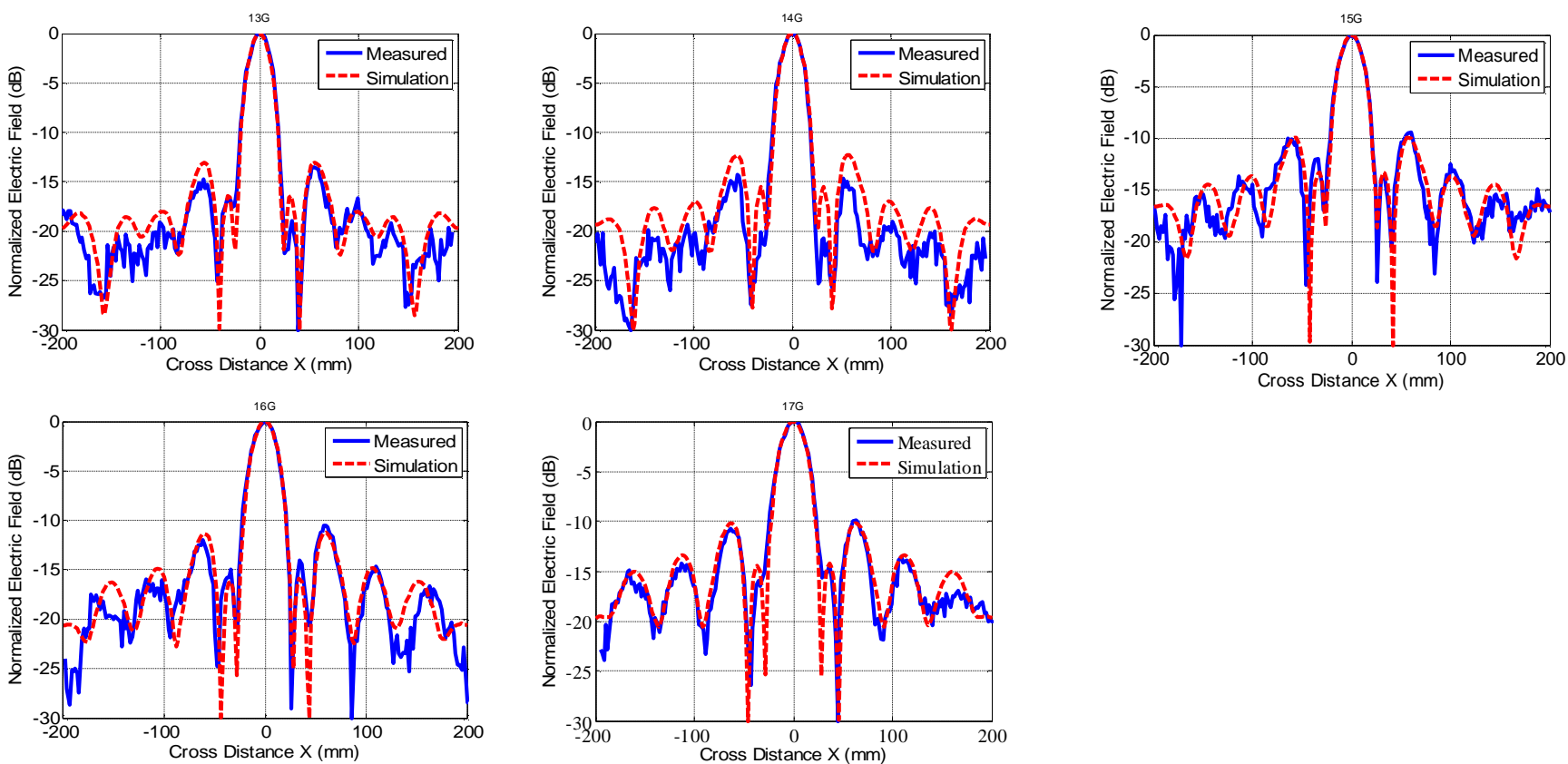

Figure 3 Normalized fields for several frequnecy points(along the $\mathrm{x}$-axis)
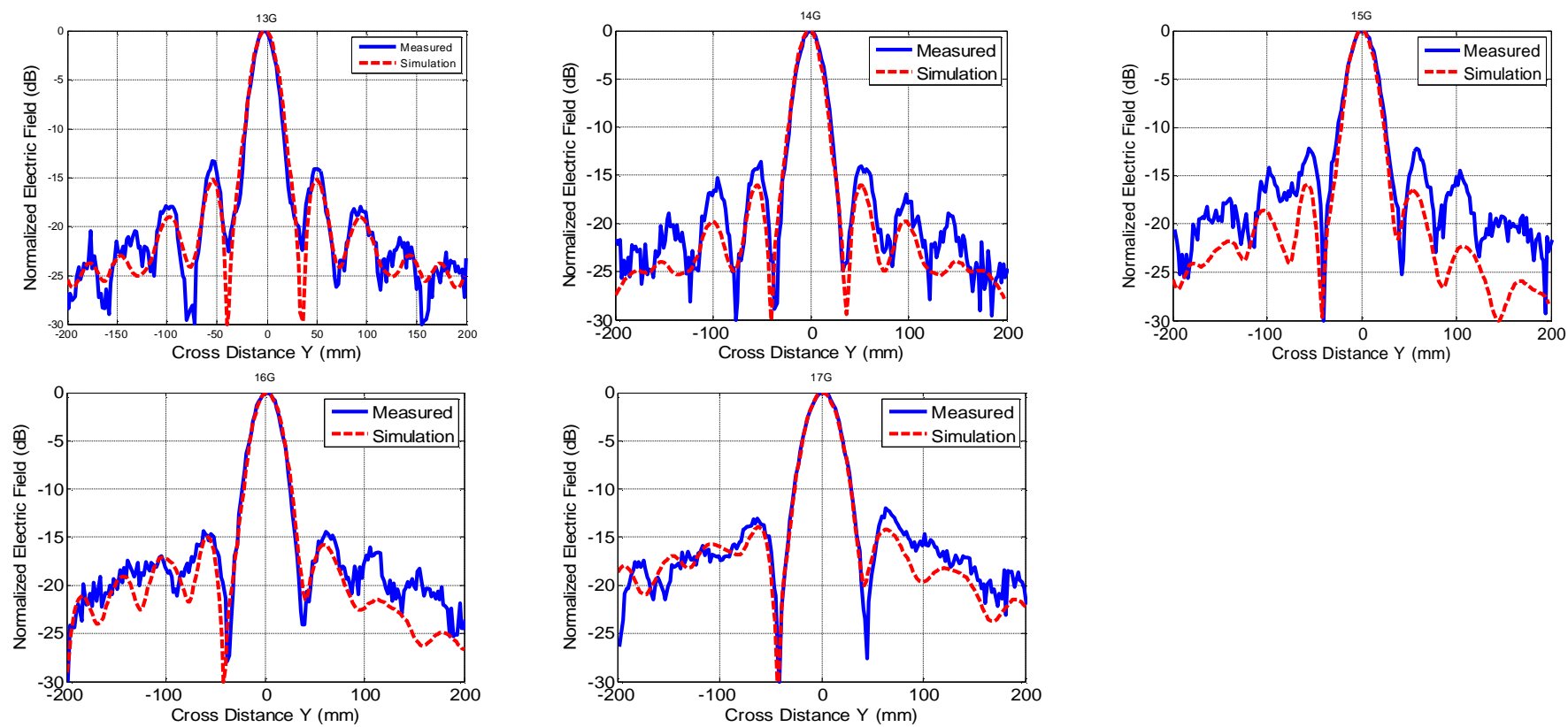

Figure 4 Normalized fields for several frequnecy points(along the y-axis)

The normalized fields of the spot are measured along the xoz-axes and yoz-axes which are shown in figure 3 and figure 4.And the measured results are compared with the simulation results in figure 3 and figure 4.From figure 3 and figure 4,we can see that measured results and simulation results are coinsident. the $\mathrm{W}_{3 \mathrm{~dB}}$ and the sidelobe level of the spot are getted from figure 3 and figure 4 which are presented in table 1.The simulation results are in agreement in the measured results. 
TABLE 1

\begin{tabular}{|c|c|c|c|c|c|c|c|c|}
\hline \multirow{2}{*}{ Freq(Ghz) } & \multicolumn{2}{|c|}{$\mathrm{W}_{3 \mathrm{dd} \_} \mathrm{X}(\mathrm{mm})$} & \multicolumn{2}{c|}{ SLL_X(dB) } & \multicolumn{2}{c|}{$\mathrm{W}_{3 \mathrm{~dB} \_} \mathrm{Y}(\mathrm{mm})$} & \multicolumn{2}{c|}{ SLL_Y(dB) } \\
\cline { 2 - 9 } & simulation & Realtest & simulation & Realtest & simulation & Realtest & simulation & Realtest \\
\hline 13 & 22.2 & 23.4 & -13.0 & -13.5 & 20.6 & 21.1 & -15.2 & -13.3 \\
\hline 14 & 22.9 & 23.5 & -12.3 & -14.3 & 23.0 & 23.6 & -16.1 & -13.6 \\
\hline 15 & 25.7 & 25.8 & -13.3 & -12.0 & 25.0 & 26.6 & -15.9 & -12.2 \\
\hline 16 & 25.4 & 26.1 & -15.9 & -14.0 & 26.9 & 27.8 & -15.0 & -14.5 \\
\hline 17 & 27.6 & 28.2 & -14.3 & -14.4 & 30.8 & 31.0 & -14.0 & -12.0 \\
\hline
\end{tabular}

The field taper in the yoz -plane is larger than that in the xoz -plane, which is caused by the polarization performance of the FZP. As a result, SLL on the yoz-plane is reduced to a lower level, and the $\mathrm{W}_{3 \mathrm{dв}}$ becomes larger compared to that of the xoz-plane, as shown in table 1 . The beamwidth in the $\mathrm{x}$-direction is narrower than in the $\mathrm{y}$-direction.

\section{CONCLUSION}

In this paper we have experimentally demonstrated the viability of the formulas for the steered near-field spot of the Fresnel zone plate versus frequency.And the spot properties are vertied again.All the measured results are conicident with the simulation results.

\section{REFERENCES}

[1] Zhiping Li, Zhengpeng Wang, Jianhua Wu, and Hua Tian, "Axially Steered Spots of Near-Field Fresnel Zone Plate Lens With Sweeping Frequency" ,IEEE ANTENNAS AND WIRELESS PROPAGATION LETTERS, VOL. 13, 2014

[2] A. Douyere, J.-D. Lan Sun Luk, A. Celeste, and J.-P. Chabriat, "Modeling and simulation of a complete system of energy transmission," in IEEE Int. Symp. Antennas Propagation Dig., Jul.2006, pp. 811-814.

[3] W. C. Brown, "The history of power transmission by radio waves," IEEE Trans. Microwave Theory Tech., vol. 32, no. 2, pp. 1230-1242, Sep. 1984.

[4] S. Karimkashi and A. A. Kishk, "Focusing properties of Fresnel zone plate lens antennas in the near-field region," IEEE Trans. Antennas Propag., vol. 59, no. 5, pp. 1481-1487, May 2011.

[5] W. J. Graham, "Analysis and synthesis of axial field patterns of fo-cused apertures," IEEE Trans. Antennas Propag., vol. AP-31, no. 4,pp. 665-668, Jul. 1983. 